\title{
Physical activity and disability measures in chronic non-specific low back pain: a study of responsiveness
}

Clinical Rehabilitation 2018, Vol. 32(12) 1684-1695 (C) The Author(s) 2018 Article reuse guidelines: sagepub.com/journals-permissions DOI: $10.1177 / 0269215518787015$ journals.sagepub.com/home/cre @SAGE

\author{
Priscila K Morelhão', Márcia R Franco², \\ Crystian B Oliveira', Thalysi M Hisamatsu', \\ Paulo H Ferreira ${ }^{3}$, Leonardo OP Costa 4 , \\ Chris G Maher ${ }^{5,6}$ and Rafael Zambelli Pinto
}

\begin{abstract} were recruited. were calculated.

\footnotetext{
'Department of Physical Therapy, Faculty of Science and Technology, Sao Paulo State University (UNESP), Presidente Prudente, Brazil

2Department of Physical Therapy, Centro Universitário UNA, Contagem, Brazil

${ }^{3}$ Discipline of Physiotherapy, Faculty of Health Science, The University of Sydney, Sydney, Australia

${ }^{4}$ Masters and Doctoral Programs in Physical Therapy, Universidade Cidade de São Paulo, São Paulo, Brazil

${ }^{5}$ Sydney School of Public Health, Faculty of Medicine and Health, The University of Sydney, Sydney, Australia
}

Objectives: To compare the responsiveness of disability measures with physical activity measures in patients with chronic low back pain (CLBP) undergoing a course of physical therapy treatment.

Design: This is a prospective cohort study with two-month follow-up.

Subjects: A total of 106 patients presenting with non-specific CLBP of more than three months duration

Main measures: Disability measures investigated were Quebec Back Pain Disability Scale and Roland Morris Disability Questionnaire. Physical activity measures analyzed include the Baecke Habitual Physical Activity Questionnaire and objective measures derived from an accelerometer (i.e. total time spent in moderate-to-vigorous and light physical activity, number of steps and counts per minute). Disability and physical activity measures were collected at the baseline and after eight weeks of treatment. For the responsiveness analyses, effect size (ES) and standardized response mean (SRM) were calculated. Correlations between the change in disability and physical activity measures

Results: Responsiveness for disability measures was considered to be large with ESs ranging from -1.03 to -1.45 and SRMs ranging from -0.99 to -1.34 , whereas all physical activity measures showed values lower 
than 0.20 . Changes in disability measures did not correlate with changes in physical activity measures (correlation coefficients ranged from -0.10 to 0.09 ).

Conclusion: Disability measures were responsive after a course of physical therapy treatment in patients with CLBP. The lack of responsiveness in the physical activity measures might be due to the inability of these measures to detect change over time or the use of an intervention not designed to increase physical activity levels.

\section{Keywords \\ Low back pain, physical activity, questionnaire, responsiveness}

Date received: I5 December 20I7; accepted: 14 June 2018

\section{Introduction}

Physical functioning or the patient's ability to carry out daily activities is considered to be a core outcome for clinical trials in non-specific low back pain. ${ }^{1}$ The impact of pain on physical functioning can be expressed in terms of the patient's level of disability or physical activity. ${ }^{2}$ While these measures appear to share some similarities due to the impact of pain on daily functioning, they reflect different constructs. Disability in patients with low back pain is mainly assessed through questionnaires, such as the Roland Morris Disability Questionnaire $^{3}$ and the Quebec Back Pain Disability Scale, ${ }^{4}$ which cover a range of spinerelated functions (e.g. twisting, bending over and sitting) likely to be affected by the presence of pain in the lower back. Physical activity, however, can be measured using self-reported questionnaires (e.g. the Baecke Habitual Physical Activity Questionnaire $)^{5}$ or objective instruments such as accelerometers, ${ }^{6}$ which measure the perceived or record in real time, the amount and/or intensity of body movements during free-living activities.

Responsiveness to change or the ability of a measure to detect real change over a pre-specified time frame is an important measurement property for any measure to be used as an outcome. ${ }^{7}$ While there is evidence to support the responsiveness of disability measures, ${ }^{8-11}$ to our knowledge, studies comparing the responsiveness of disability measures with physical activity measures in patients with chronic low back pain (CLBP) are lacking.
Therefore, the objective of this study was to compare the responsiveness of disability measures with physical activity measures in patients with CLBP undergoing a course of physical therapy treatment. For the purpose of this study, physical therapy treatment was standardized to reflect the recommendations from current clinical practice guidelines for the management of CLBP, consisting of exercise therapy and education to remain active and return to normal activities as soon as possible. ${ }^{12,13}$

\section{Methods}

We conducted a prospective cohort study with twomonth follow-up. This study was approved by the research ethics committee from the São Paulo State University (CAAE: 36285414.40000.5402). All participants signed a consent form before enrolling in the study.

Consecutive patients with low back pain seeking care at an outpatient physical therapy clinic in Presidente Prudente, Brazil, were invited to participate. Participants were considered eligible if they had non-specific CLBP, defined as pain or discomfort localized between the last rib and above the inferior gluteal fold, with or without referred pain to the leg, presenting with symptoms of at least three months' duration and aged between 18 and 60 years. In addition, patients should have obtained a score of at least moderate on question 7 ("How much bodily pain have you had during the past week?") or 8 ("During the past week, how much did pain 
interfere with your normal work, including both work outside the home and housework?") of the SF36 questionnaire. ${ }^{14}$ This criterion was applied in order to include participants who were representative of a patient population with low back pain seeking physical therapy treatment. Patients with common imaging findings, such as osteoarthritis, grade I spondylolysis and spondylolisthesis or protrusion/herniation/prolapsed disc, but with clinical symptoms that met the criteria for inclusion/exclusion, were considered eligible. Subjects were excluded if they had known or suspected serious spinal pathology such as fracture, metastatic, inflammatory or infective diseases of the spine, cauda equina syndrome, radiculopathy (at least two of the following signs: weakness, reflex change or sensation loss, associated with the same spinal nerve) and any contraindication to physical exercise according to the guidelines of the American College of Sports Medicine. ${ }^{15}$

Patients were treated at a local outpatient physical therapy clinic with a group exercise program. The outpatient physical therapy clinic is available to patients from the local community free of charge. Treating physical therapists were instructed to follow the clinical practice guidelines for the management of CLBP which recommends exercise therapy and education to remain active and return to normal activities, including early return to work, as soon as possible. ${ }^{12,13}$ The group exercise program consisted of one-hour group sessions twice a week for two months. Each session started with a program of progressive or graded exercise and finished with an education messages to stay active (i.e. avoid bed rest) and gradually resume normal activities in the form of "Tip of the Day." The exercise components included warm-up (i.e. 5 to 10 -min walking) and stretching, up to 10 individual exercises (combining core stabilization exercises for lumbo-pelvic region and strengthening exercise for upper and lower limbs), cool down and relaxation. The exercises were progressed every one or two weeks by increasing the number of sets and repetitions and/or making the exercises more challenging.

To characterize the sample, we collected at baseline: anthropometric and demographic data (i.e. age, body mass index, education level and work status), pain duration, pain intensity in the last 24 hours measures with a $0-10$ numerical pain rating scale, ${ }^{16}$ fear of movement measured with FearAvoidance Beliefs Questionnaire, ${ }^{17}$ and depression measured with the Beck Depression Inventory. ${ }^{18}$

Disability and physical activity measures were assessed at baseline and at two-month follow-up. Disability measures used were the Roland Morris Disability Questionnaire ${ }^{3}$ and Quebec Back Pain Disability Scale. ${ }^{4}$ The Roland Morris Disability Questionnaire consists of 24 yes/no items with a total score ranging from 0 (no disability) to 24 (maximal disability). ${ }^{3}$ The Quebec Back Pain Disability Scale consists of 20 items scored on a $0-5$ scale with a total score ranging from 0 (no disability) to 100 (maximum disability). ${ }^{4}$

Physical activity levels were measured with two assessment methods: self-reported and objective measures. The Baecke Physical Activity Questionnaire $^{5}$ records self-reported physical activity levels within a typical week. This questionnaire consists of 16 items assessing different domains of physical activity (i.e. work, sports, leisure time). The total score varies between 3 and 15 with a higher score indicating a higher physical activity level. This questionnaire has shown acceptable reliability but only fair correlation with accelerometer data (i.e. number of steps per day and vector magnitude counts per minutes) in patients with CLBP. ${ }^{19}$ The objective physical activity measures were collected using the ActiGraph GT3X-BT (ActiGraph, LLC, Pensacola, FL, USA) which is a non-invasive, small, lightweight triaxial accelerometer $(4.6 \mathrm{~cm} \times 3.3 \mathrm{~cm} \times 1.5 \mathrm{~cm}, 19 \mathrm{~g}) .{ }^{20}$ Patients were told to wear the device during waking hours for seven consecutive days, at baseline and at twomonth follow-up, on the right hip fastened by an elastic waistband. ${ }^{21}$ Participants were asked not to wear the accelerometer during certain activities such as swimming, bathing and sleep. Participants received short message service reminders to wear the accelerometer each morning at a pre-determined time chosen by the own participant. For the purpose of this study, acceleration data were sampled at $30 \mathrm{~Hz}$ and analyzed in 60 -second epochs. Accelerometer data were analyzed and computed 
with ActiLife 6 software (ActiGraph, LLC). For each patient, a complete data set was defined as at least 10 hours per day of monitored wear during at least five days. Non-wear periods were defined as time intervals of at least 60 consecutive minutes of zero counts, with an activity interruption allowance of $0-100$ counts/min lasting a maximum of 2 consecutive minutes. ${ }^{22}$ Outcome measures for each participant were as follows: (1) total time spent in moderate-to-vigorous physical activity per day, (2) light physical activity per day, (3) total steps per day and (4) counts per minute. The outcome measures were obtained from the vertical axis. Moderate-to-vigorous physical activity was defined as values greater than 1952 counts/min and light physical activity was defined as values between 100 and 1951 counts/min. ${ }^{23}$ Counts per minute were calculated by dividing the sum of activity counts of the vertical axis by the number of valid minutes. ${ }^{23}$

Descriptive statistics were calculated for all measures at baseline assessment using mean and $\mathrm{SD}$, median and interquartile range (IQR) and frequency and proportion depending on data distribution.

Responsiveness was calculated for disability and physical activity measures. Responsiveness or the ability of a measure to detect real change in physical activity levels over pre-specified time frame was calculated using the effect size (Effect Size = Mean follow-up minus Mean baseline/SD of baseline) and the standardized response mean (SRM) (Standardized Response Mean $=$ Mean follow-up minus Mean baseline/ SD of the change). ${ }^{7}$ For Effect Size and SRM, we calculated confidence intervals of $84 \% .{ }^{24}$ The $84 \%$ confidence interval was used because nonoverlapping $84 \%$ confidence intervals are equivalent to a $Z$ test of means at the 0.05 level. ${ }^{24}$ Effect size and SRM should be interpreted using the following benchmarks: an effect size of 0.2 being considered small, 0.5 as medium and 0.8 or greater as large. ${ }^{25}$

For the correlation analysis, we used Spearman's rho to investigate the correlation between the change in disability measures and the change detected by the Baecke Physical Activity
Questionnaire and accelerometers variables (i.e. counts per minutes, steps per day, moderate-tovigorous physical activity per day and light physical activity per day). Scatter plots were also used to visualize the relationship between these measures. The correlation was interpreted using the following cut-offs: 0.00 to 0.25 little or no relationship; 0.25 to 0.50 fair relationship; 0.50 to 0.75 moderate to good relationship; and above 0.75 good to excellent relationship. ${ }^{26}$ We hypothesized that the changes in disability and physical activity measures would show fair relationship given that a meta-analysis of cross-sectional data had previously reported a moderate negative correlation $(r=-0.33)$ between these measures. ${ }^{2}$

The responsiveness of the physical activity and disability measures was investigated using a priori-formulated hypotheses following recommendations according to Mokkink et al. ${ }^{27}$ Based on the results of previous studies, ${ }^{9,28}$ we expected at least medium responsiveness for disability measures. For physical activity measures, we hypothesized that in patients with CLBP the responsiveness would vary from small to medium as in previous studies with sedentary adults. ${ }^{29}$

\section{Results}

From September 2014 to October 2016, a total of 106 patients completed the two-month intervention program and were included in the analyses. Figure 1 shows the flow of participants in the study. The baseline characteristics of the 106 patients who completed the study are described in Table 1.

Responsiveness analyses for disability and physical activity measures are provided in Table 2 . Disability measures were more responsiveness compared to physical activity measures. For Roland Morris Disability Questionnaire and Quebec Back Pain Disability Scale, effect sizes and SRMs showed large effects. For all physical activity measures, effect sizes and SRMs were lower than 0.20 indicating that physical activity measures were not able to detect real change in physical activity levels over time. In addition, the correlation between the change in disability and physical activity measures were all non-significant 


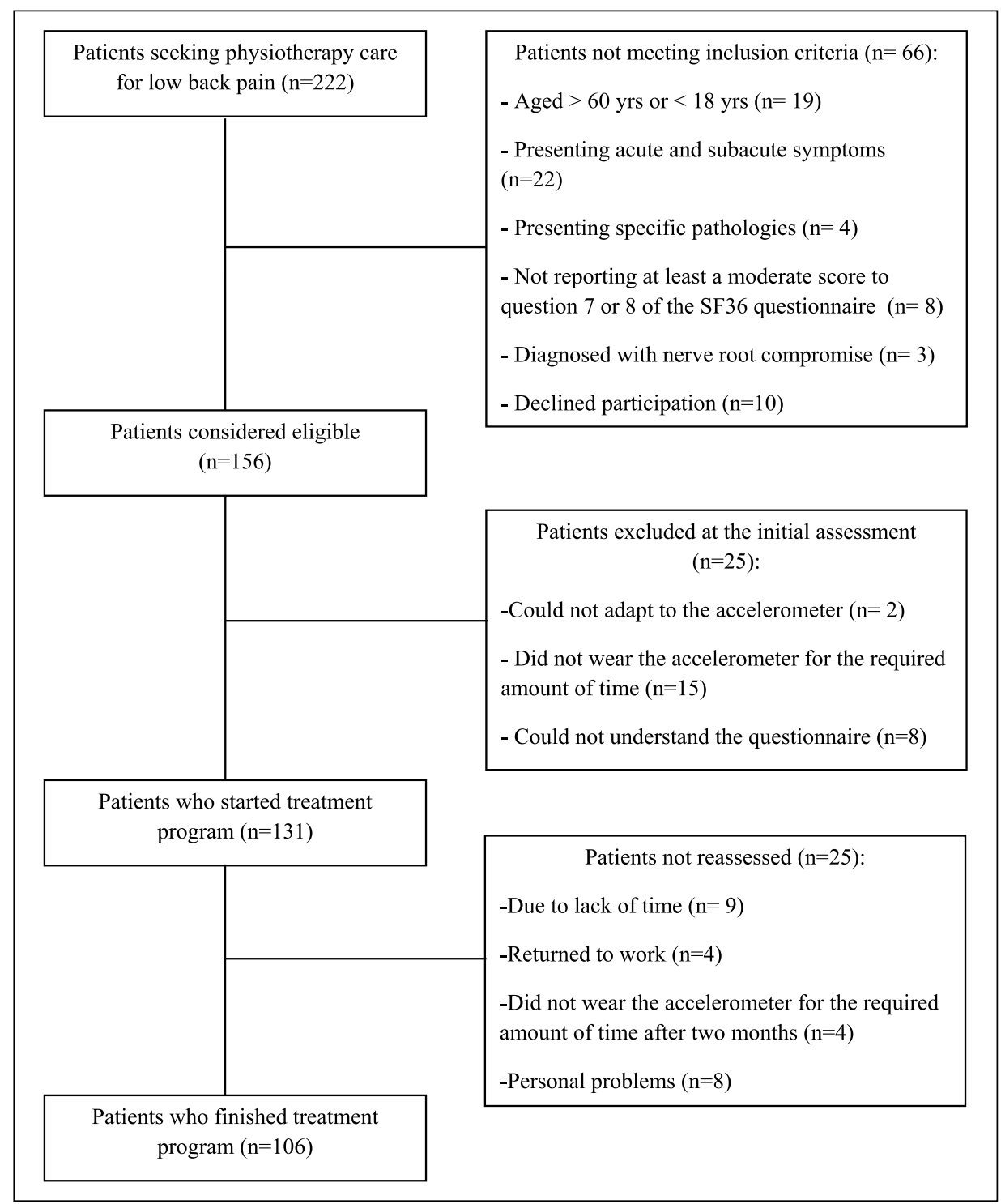

Figure I. Flow diagram of participants in the study.

(Table 3). The lack of relationship between these measures can also be seen by visual inspection of scatter plots (Figure 2).

\section{Discussion}

Our findings revealed that both disability measures were able to detect change over time, whereas all physical activity measures were not responsive after a course of physical therapy treatment based on guideline-endorsed intervention for CLBP. Our findings also showed that the type of assessment method used to measure physical activity levels did not influence the lack of responsiveness.

A strength of this study was the use of a large sample size $(n=106)$. According to the COnsensusbased Standards for the selection of health Measurement Instruments, a sample size of at least 
Table I. Demographics and clinical characteristics of patients.

\begin{tabular}{lc}
\hline Characteristics & Total sample $(n=106)$ \\
\hline Age $($ years) & $40(1 \mathrm{I} .60)$ \\
BMI $\left(\mathrm{kg} / \mathrm{m}^{2}\right)$ & $27.04(5.92)$ \\
Female, $\mathrm{n}(\%)$ & $59(69)$ \\
Symptoms duration (months) & $12(6-48)$ \\
Work status, $\mathrm{n}(\%)$ & \\
Employed full time & $47(44.3)$ \\
Employed part time & $18(17.0)$ \\
Unemployed & $41(38.7)$ \\
Education, $\mathrm{n}(\%)$ & \\
No education beyond primary schoola & $18(11.7)$ \\
No education beyond secondary schoolb & $44(28.6)$ \\
Tertiary education incomplete & $21(13.6)$ \\
Tertiary education complete & $23(14.9)$ \\
FABQ (0-96) & $43.96(19.54)$ \\
BDI (0-63) & $10.77(6.95)$ \\
Pain $(0-10)$ & $6.30(1.80)$ \\
\hline
\end{tabular}

BMI: body mass index; FABQ: Fear avoidance beliefs questionnaire; BDI: Beck depression inventory.

Data are mean (SD), median (interquartile range) or frequency (proportion).

aln Brazil, primary schools provide education from the age of 5 to II.

bln Brazil, secondary schools provide education from the age of 12 to 17.

100 patients is needed to rate a study as excellent with regard to the appropriateness of sample size. ${ }^{27}$ Only $16 \%$ of the eligible patients did not start or were not included in the analyses due to poor compliance with the wearing protocol. Previous studies in this area have reported a considerably higher rate of non-compliance. ${ }^{30,31} \mathrm{We}$ would argue that the strategy adopted by this study to send short message service reminder to patients might have enhanced compliance with the wearing protocol. Nevertheless, a limitation of this study was the overall dropout from the study. A total of $19 \%$ of the sample started treatment program but did not finish due to several reasons (Figure 1). Another limitation is that our findings cannot be generalized to patients with acute and subacute low back pain.

Previous studies investigating the ability of disability measures to detect change over time in a similar population have reported moderate to large responsiveness. ${ }^{8-11}$ We would argue that our results are consistent with these previous studies. Few studies, however, have investigated the responsiveness of physical activity measures in other populations..$^{29,32,33}$
A previous study investigating the responsiveness of objective physical activity measures derived from ActiGraph accelerometers in children and adolescents population found large responsiveness $(\mathrm{SRM}>0.80)$ for time spent in moderate-to-vigorous physical activity and light physical activity. ${ }^{32}$ The large responsiveness found might be due to the difference in the methodologies. While in our study we investigated the responsiveness after a course of twomonth physical therapy intervention, in the previous study, the responsiveness was assessed in a laboratory setting comparing the patients' performance while exercising in two different intensities, low versus high-intensity exercises. Another study investigated the responsiveness of objective physical activity measures derived from the ActiGraph accelerometer and two other self-report questionnaires (i.e. Australia Active Survey and US National Health Interview Survey) in diabetes population. ${ }^{33}$ The responsiveness results found for all physical activity measures were considered to be small, but those for accelerometer data were slightly higher than those for self-report questionnaires. ${ }^{33}$ Similarly, another 


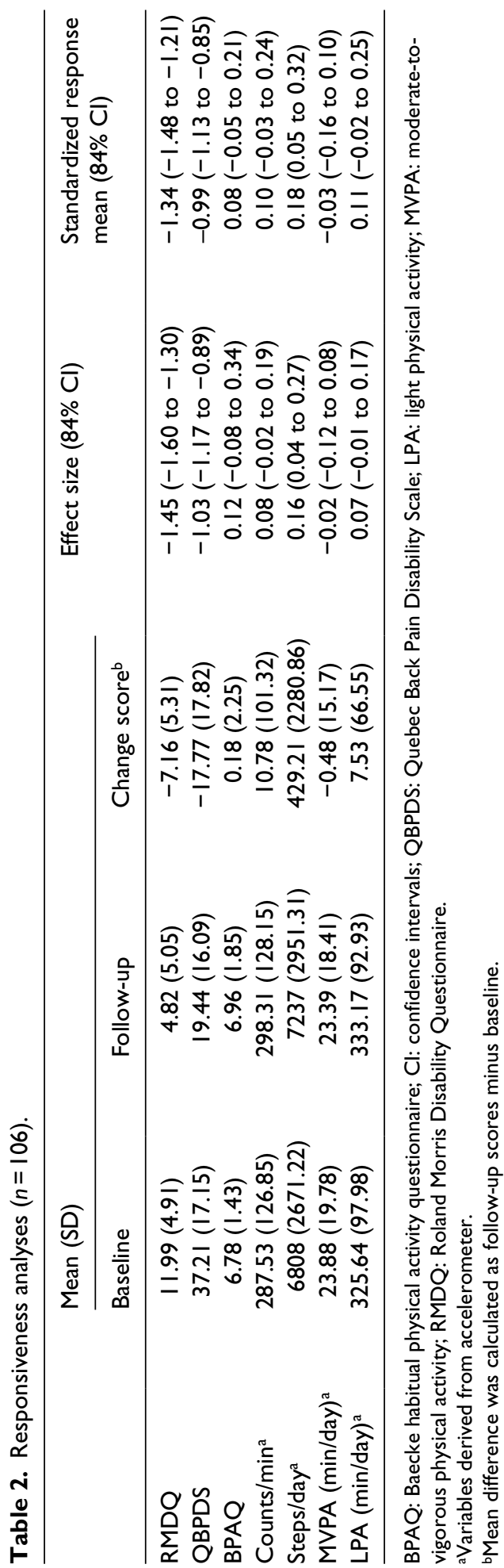

Table 3. Correlation between the changes in disability, measured with two scales, with physical activity measures.

\begin{tabular}{lrc}
\hline Measurements & \multicolumn{1}{c}{ RM } & QBPDS \\
\hline Total BPAQ & 0.09 & -0.10 \\
Counts/min & 0.01 & 0.05 \\
Steps $/$ day $^{\mathrm{a}}$ & -0.04 & -0.04 \\
MVPA (min/day) & 0.08 & -0.002 \\
LPA (min/day) & -0.04 & 0.01
\end{tabular}

BPAQ: Baecke habitual physical activity questionnaire; MVPA: moderate-to-vigorous physical activity; LPA: light physical activity; RMDQ: Roland Morris Disability Questionnaire QBPDS: Quebec Back Pain Disability Scale.

a $V$ ariables derived from accelerometer.

study evaluated the responsiveness of objective physical activity measures derived from ActiGraph also found small responsiveness to change in sedentary adults. ${ }^{29}$ In contrast, our findings showed even smaller values than these previous studies. ${ }^{29,33}$ While the exercise therapy used in this study requires the patient to exercise during the treatment session only, the interventions tested in these previous studies ${ }^{29,33}$ were designed to disrupt sedentary behavior and increase physical activity levels which might result in a physical activity behavior change.

There are two possible explanations for the lack of responsiveness in the physical activity measures. One possible explanation is that the patients in this study changed their level of physical activity but the physical activity measures investigated failed to detect this change. Given that the accelerometers are influenced by the choice of data collection and processing criteria, ${ }^{34,35}$ it is possible that the criteria used in this study which has been validated for healthy adults ${ }^{23}$ were not able to capture the change in patients with CLBP. Future studies should investigate whether the use of different processing criteria, such as accelerometer cut-off points to quantify physical activity levels or energy expenditure algorithms that combine information from all three axes (i.e. vector magnitude counts), influence the responsiveness of physical activity measures. Regarding the Baecke Physical Activity Questionnaire, this is a self-reported tool developed for epidemiological studies ${ }^{5}$ which may not be appropriate to detect change after a short period. 


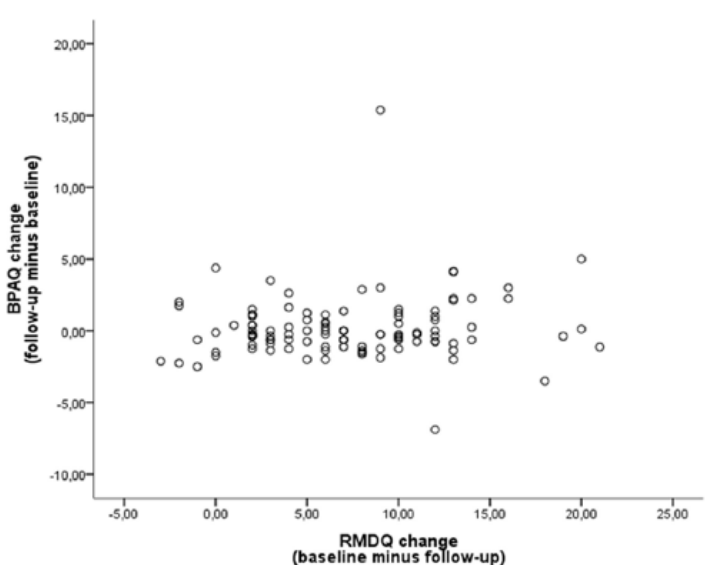

(a)

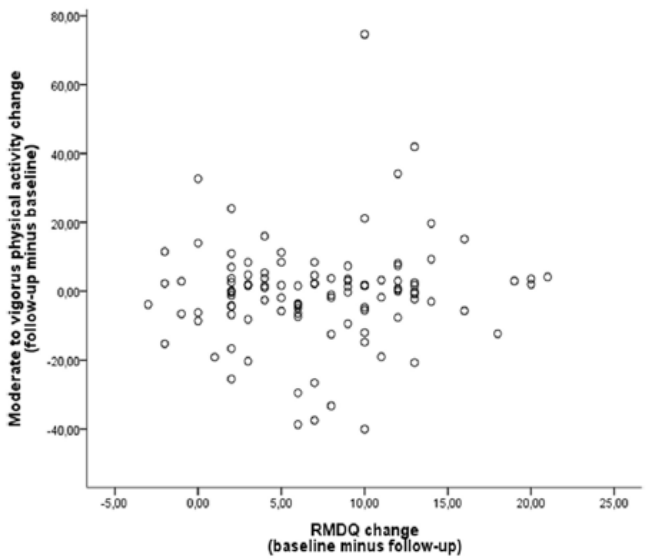

(c)

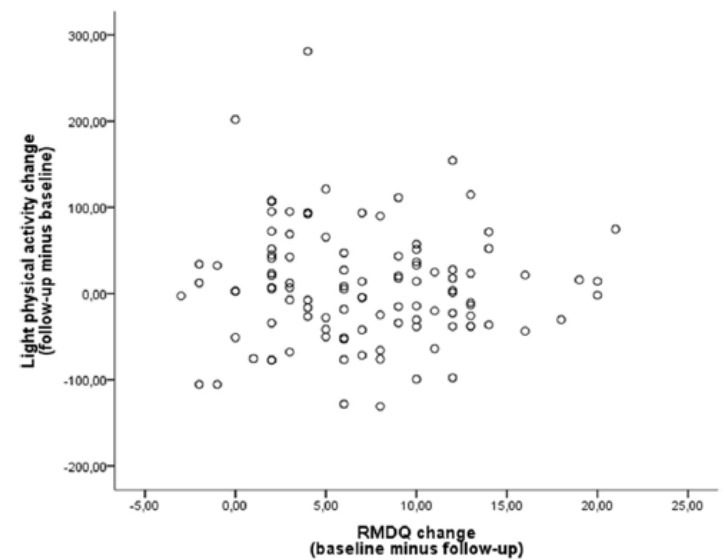

(e)

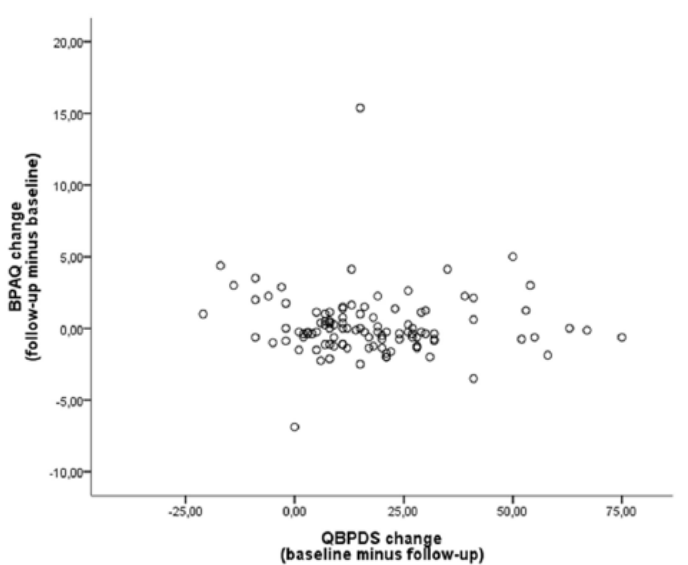

(b)

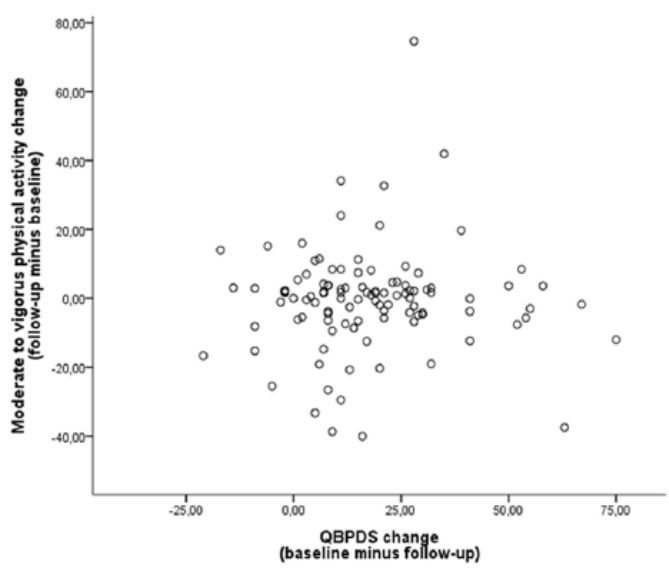

(d)

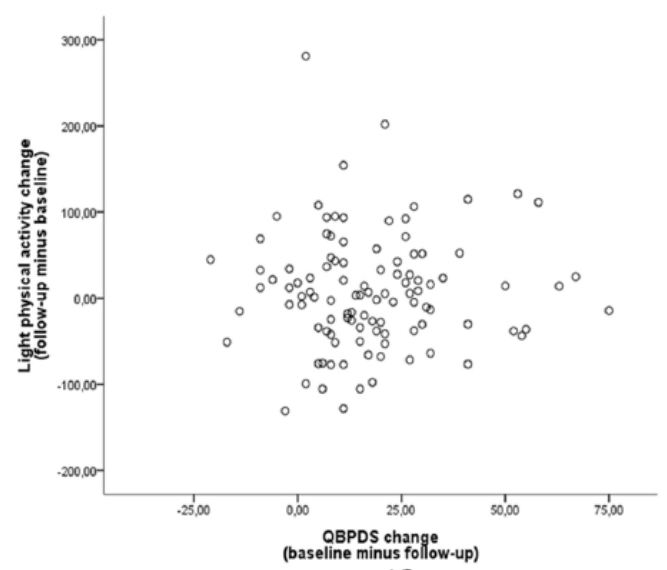

(f)

Figure 2. (Continued) 


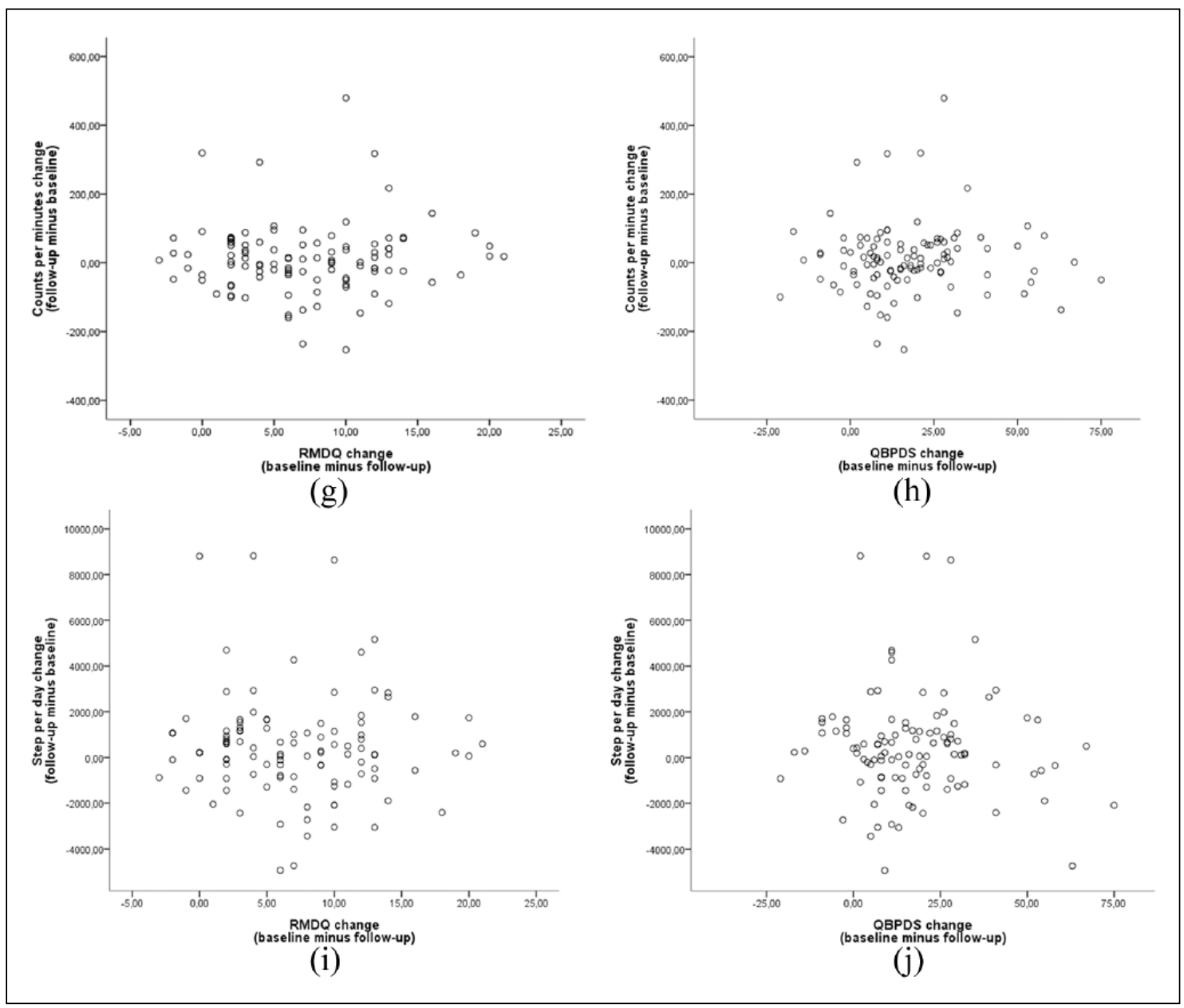

Figure 2. Scatter plots showing correlation between changes in (a) Baecke Physical Activity Questionnaire (BPAQ) and Roland Morris Disability Questionnaire (RMDQ) scale, (b) BPAQ and Quebec Back Pain Disability Scale (QBPDS), (c) moderate-to-vigorous physical activity (MVPA) and RMDQ, (d) MVPA and QBPDS, (e) light physical activity change (LPA) and RMDQ, (f) LPA and QBPDS, (g) counts per minute and RMDQ, (h) counts per minute and QBPDS, (i) step per day and RMDQ and (j) step per day and QBPDS.

Another possible explanation is that the guidelineendorsed intervention administered in this study was effective for improving the patients' level of functional activities or the ability to perform spinerelated activities such as twisting and bending over but failed to increase the level of physical activity. This finding is not in accordance with the assumption that after a course of treatment patients would present with lower levels of pain and disability and consequently become more physically active. In this case, the guideline-endorsed intervention used in this study makes patients more active during the treatment session but is not designed to make them more physically active during their daily life. In fact, a recent systematic review published by our research group shows that the available physical activity-based interventions failed to increase physical activity levels in people with chronic musculoskeletal pain..$^{36}$ We would argue that to change patients' behavior toward a more active lifestyle, 
interventions designed specifically for this purpose are needed. Nevertheless, since the publication of this systematic review, we identified at least four randomized controlled trials investigating the efficacy of interventions specifically designed to improve physical activity levels in patients with CLBP. Of these, one published trial ${ }^{37}$ used a selfreported physical activity measure as an outcome but did not provide information on disability measures and three trial protocols ${ }^{38-40}$ collecting concomitant information on self-reported and/or objective physical activity and disability measures. The results of these trials will help understanding the value of this type of intervention on physical activity measures as well as on clinical outcome, such as disability and pain intensity.

Physical therapy as a primary care profession is now facing the challenge to take a more active role to promote physical activity at the individual, societal and policy levels ${ }^{41}$ because physical therapists are considered to be well-positioned to provide specific education about physical activity during individual therapeutic intervention and to deliver evidence-based intervention about active lifestyle in community-based primary care initiatives. ${ }^{42}$ This may even be more relevant to patients with CLBP due to the high prevalence of comorbidities such as cardiovascular disease, ${ }^{43,44}$ diabetes ${ }^{45}$ and obesity. ${ }^{46}$ As physical activity promotion has important implications for the management and prevention of these comorbidities, physical activity measures with appropriate measurement properties are needed to assist physical therapists assessing and monitoring their patients' physical activity levels. We would encourage future studies to test other accelerometer data processing criteria or develop new instruments that are responsive to changes in physical activity levels during free-living activities in patients with CLBP. Given that physical activity is central to achieving better health back ${ }^{47}$ but current physical activity-based interventions in this area are not specifically designed to increase physical activity levels, ${ }^{36}$ we would also recommend future studies to test the responsiveness of physical activity measures with interventions that also incorporate strategies for changing physical activity behavior, including provision of instruction, action planning and reinforcing effort toward behavior. ${ }^{48}$

\section{Clinical messages}

- Self-reported and objective physical activity measures showed poor responsiveness to a guideline-endorsed physical therapy intervention for chronic low back pain compared with disability measures.

- The lack of responsiveness found for physical activity measures suggests that available physical activity measures are not able to capture the change in physical activity levels or current guidelineendorsed physical therapy intervention is not able to improve patients' physical activity level.

\section{Declaration of conflicting interests}

The author(s) declared no potential conflicts of interest with respect to the research, authorship and/or publication of this article.

\section{Funding}

The author(s) disclosed receipt of the following financial support for the research, authorship, and/or publication of this article: R.Z.P., P.K.M. and T.M.H. were supported by the Sao Paulo Research Foundation (grant numbers: 2014/14077-8, 2015/02744-2, 2016/04113-2). C.G.M. is supported by a principal research fellowship from the National Health and Medical Research Council of Australia.

\section{ORCID iD}

Rafael Zambelli Pinto (iD https://orcid.org/0000-00022775-860X

\section{References}

1. Chiarotto A, Deyo RA, Terwee CB, et al. Core outcome domains for clinical trials in non-specific low back pain. Eur Spine J 2015; 24: 1127-1142.

2. Lin CW, McAuley JH, Macedo L, et al. Relationship between physical activity and disability in low back pain: a systematic review and meta-analysis. Pain 2011; 152: 607-613.

3. Roland M and Morris R. A study of the natural history of back pain. Part I: development of a reliable and sensitive measure of disability in low-back pain. Spine 1983; 8: 141-144. 
4. Kopec JA, Esdaile JM, Abrahamowicz M, et al. The Quebec Back Pain Disability Scale. Measurement properties. Spine 1995; 20: 341-352.

5. Baecke JA, Burema $\mathrm{J}$ and Frijters JE. A short questionnaire for the measurement of habitual physical activity in epidemiological studies. Am J Clin Nutr 1982; 36: 936-942.

6. Trost SG and O'Neil M. Clinical use of objective measures of physical activity. Br J Sports Med 2014; 48: 178-181.

7. Husted JA, Cook RJ, Farewell VT, et al. Methods for assessing responsiveness: a critical review and recommendations. J Clin Epidemiol 2000; 53: 459-468.

8. Zerkak D, Metivier JC, Fouquet B, et al. Validation of a French version of Roland-Morris questionnaire in chronic low back pain patients. Ann Phys Rehabil Med 2013; 56: 613-620.

9. Wilhelm F, Fayolle-Minon I, Phaner V, et al. Sensitivity to change of the Quebec Back Pain Disability Scale and the Dallas Pain Questionnaire. Ann Phys Rehabil Med 2010; 53: 15-23.

10. Lauridsen HH, Hartvigsen J, Manniche C, et al. Responsiveness and minimal clinically important difference for pain and disability instruments in low back pain patients. BMC Musculoskelet Disord 2006; 7: 82.

11. Chapman JR, Norvell DC, Hermsmeyer JT, et al. Evaluating common outcomes for measuring treatment success for chronic low back pain. Spine 2011; 36: S54-S68.

12. O'Connell NE, Cook CE, Wand BM, et al. Clinical guidelines for low back pain: a critical review of consensus and inconsistencies across three major guidelines. Best Pract Res Clin Rheumatol 2016; 30: 968-980.

13. Almeida M, Saragiotto B, Richards B, et al. Primary care management of non-specific low back pain: key messages from recent clinical guidelines. Med J Aust 2018; 208: 272-275.

14. Ciconelli RM, Ferraz MB, Santos W, et al. Tradução para a língua portuguesa e validação do questionário genérico de avaliação de qualidade de vida SF-36 (Brasil SF-36). Rev Bras Reumatol 1999; 39: 143-150.

15. American College of Sports Medicine. ACSM's guidelines for exercise testing and prescription. 5th ed. Baltimore, MD: Lippincott Williams \& Wilkins, 2006.

16. Ross RLP. Clinical assessment of pain. In: van Dieen JH (ed.) Assessment in occupational therapy and physical therapy. Philadelphia, PA: WB Saunders, 1997, pp.123-133.

17. Waddell G, Newton M, Henderson I, et al. A FearAvoidance Beliefs Questionnaire (FABQ) and the role of fear-avoidance beliefs in chronic low back pain and disability. Pain 1993; 52: 157-168.

18. Beck AT, Ward CH, Mendelson M, et al. An inventory for measuring depression. Arch Gen Psychiatry 1961; 4: 561-571.

19. Carvalho FA, Morelhão PK, Franco MR, et al. Reliability and validity of two multidimensional selfreported physical activity questionnaires in people with chronic low back pain. Musculoskeletal Sci Pract 2017; 27: $65-70$.
20. Matthews CE, Chen KY, Freedson PS, et al. Amount of time spent in sedentary behaviors in the United States, 2003-2004. Am J Epidemiol 2008; 167: 875-881.

21. Tudor-Locke C, Barreira TV and Schuna JM Jr. Comparison of step outputs for waist and wrist accelerometer attachment sites. Med Sci Sports Exerc 2015; 47: 839-842.

22. Troiano RP, Berrigan D, Dodd KW, et al. Physical activity in the United States measured by accelerometer. Med Sci Sports Exerc 2008; 40: 181-188.

23. Freedson PS, Melanson E and Sirard J. Calibration of the Computer Science and Applications, Inc. accelerometer. Med Sci Sports Exerc 1998; 30: 777-781.

24. Tryon WW. Evaluating statistical difference, equivalence, and indeterminacy using inferential confidence intervals: an integrated alternative method of conducting null hypothesis statistical tests. Psychol Methods 2001; 6: 371-386.

25. Cohen J. Statistical power analysis for the behavioral sciences L. 2nd ed. Hillsdale, NJ: Lawrence Erlbaum Associates, 1988.

26. Portney L and Watkins M. Foundations of clinical research: applications to practice. 2 nd ed. Upper Saddle River, NJ: Prentice Hall, 2000.

27. Mokkink LB, Terwee CB, Patrick DL, et al. The COSMIN checklist manual, http://www.cosmin.nl (2009, accessed August 2017).

28. Macedo LG, Maher CG, Latimer J, et al. Responsiveness of the 24-, 18- and 11-item versions of the Roland Morris Disability Questionnaire. Eur Spine J 2011; 20: 458-463.

29. Swartz AM, Rote AE, Cho YI, et al. Responsiveness of motion sensors to detect change in sedentary and physical activity behaviour. Br J Sports Med 2014; 48: 1043-1047.

30. Hurley DA, Tully MA, Lonsdale C, et al. Supervised walking in comparison with fitness training for chronic back pain in physiotherapy: results of the SWIFT singleblinded randomized controlled trial (ISRCTN17592092). Pain 2015; 156: 131-147.

31. McDonough SM, Tully MA, Boyd A, et al. Pedometerdriven walking for chronic low back pain: a feasibility randomized controlled trial. Clin J Pain 2013; 29: 972-981.

32. Montoye AH, Pfeiffer KA, Suton D, et al. Evaluating the responsiveness of accelerometry to detect change in physical activity. Meas Phys Educ Exerc Sci 2014; 18 : 273-285.

33. Lee WY, Clark BK, Winkler E, et al. Responsiveness to change of self-report and device-based physical activity measures in the living well with diabetes trial. J Phys Act Health 2015; 12: 1082-1087.

34. Migueles JH, Cadenas-Sanchez C, Ekelund U, et al. Accelerometer data collection and processing criteria to assess physical activity and other outcomes: a systematic review and practical considerations. Sports Med 2017; 47 : 1821-1845.

35. Smith MP, Standl M, Heinrich J, et al. Accelerometric estimates of physical activity vary unstably with data handling. PLoS ONE 2017; 12: e0187706. 
36. Oliveira CB, Franco MR, Maher CG, et al. Physical activity interventions for increasing objectively measured physical activity levels in chronic musculoskeletal pain: systematic review. Arthritis Care Res 2016; 68: 1832-1842.

37. Schaller A, Dintsios CM, Icks A, et al. Promoting physical activity in low back pain patients: six months follow-up of a randomised controlled trial comparing a multicomponent intervention with a low intensity intervention. Clin Rehabil 2016; 30: 865-877.

38. Milosavljevic S, Clay L, Bath B, et al. Walking away from back pain: one step at a time - a community-based randomised controlled trial. BMC Public Health 2015; 15: 144.

39. Amorim AB, Pappas E, Simic M, et al. Integrating Mobile health and Physical Activity to reduce the burden of Chronic low back pain Trial (IMPACT): a pilot trial protocol. BMC Musculoskelet Disord 2016; 17: 36.

40. Oliveira CB, Franco MR, Maher CG, et al. The efficacy of a multimodal physical activity intervention with supervised exercises, health coaching and an activity monitor on physical activity levels of patients with chronic, nonspecific low back pain (Physical Activity for Back Pain (PAyBACK) trial): study protocol for a randomised controlled trial. Trials 2018; 19: 40.

41. Parra DC, Bradford ECH, Clark BR, et al. Population and community-based promotion of physical activity: a priority for physical therapy. Phys Ther 2017; 97: 159-160.
42. Frerichs W, Kaltenbacher E, van de Leur JP, et al. Can physical therapists counsel patients with lifestyle-related health conditions effectively? A systematic review and implications. Physiother Theory Pract 2012; 28: 571-587.

43. Ha IH, Lee J, Kim MR, et al. The association between the history of cardiovascular diseases and chronic low back pain in South Koreans: a cross-sectional study. PLOS ONE 2014; 9: e93671.

44. Ryan CG, McDonough S, Kirwan JP, et al. An investigation of association between chronic musculoskeletal pain and cardiovascular disease in the Health Survey for England (2008). Eur J Pain 2014; 18: 740-750.

45. Hoff OM, Midthjell K, Zwart JA, et al. The association between diabetes mellitus, glucose, and chronic musculoskeletal complaints. Results from the Nord-Trondelag Health Study. BMC Musculoskelet Disord 2008; 9: 160.

46. Shiri R, Karppinen J, Leino-Arjas P, et al. The association between obesity and low back pain: a meta-analysis. Am J Epidemiol 2010; 171: 135-154.

47. O'Keeffe M, Maher CG and O'Sullivan K. Unlocking the potential of physical activity for back health. Br J Sports Med 2017; 51: 760-761.

48. Williams SL and French DP. What are the most effective intervention techniques for changing physical activity self-efficacy and physical activity behaviour - and are they the same? Health Educ Res 2011; 26: 308-322. 